\title{
Accurate Outage Probability Evaluation of Equal Gain Combining Receivers
}

\author{
Nadhir Ben Rached, Abla Kammoun, Mohamed-Slim Alouini, and Raul Tempone \\ Computer, Electrical and Mathematical Science and Engineering (CEMSE) Division \\ King Abdullah University of Science and Technology (KAUST) \\ Thuwal, Makkah Province, Saudi Arabia \\ \{nadhir.benrached, abla.kammoun, slim.alouini, raul.tempone\}@kaust.edu.sa
}

\begin{abstract}
We consider the evaluation of the outage probability (OP) for $L$-branch equal gain combining diversity receivers operating over fading channels, i.e. equivalently the cumulative distribution function (CDF) of the sum of the $L$ channel envelopes. Generally, closed-form expressions of the $O P$ values are out of reach. Moreover, the use of naive Monte Carlo (MC) simulations is not a good alternative since it is expensive in terms of number of samples when small values of OP are considered. In this paper, we use the concept of importance sampling (IS), being known to yield accurate estimates using few number of simulations runs. The proposed IS scheme is essentially based on sample rejection where the IS probability density function (PDF) is the truncation of the underlying PDF over the $L$ dimensional sphere. It assumes the knowledge of the CDF of the sum of the $L$ channel gains in a closed-form expression. Such an assumption is not restrictive since it holds for various challenging fading models. As an illustration, we apply the proposed estimator to the cases of independent Rayleigh, correlated Rayleigh, and independent and identically distributed Rice fading channels and prove that it achieves the well-desired bounded relative error property. Finally, we validate these theoretical results through some selected experiments.
\end{abstract}

Index Terms-Outage probability, equal gain combining, importance sampling, sample rejection, bounded relative error.

\section{INTRODUCTION}

Sums of random variables (RVs) occur in many challenging wireless communication applications. For instance, the instantaneous signal-to-noise-ratio (SNR) expressions at the output of equal gain combining (EGC) and maximum ratio combining (MRC) diversity receivers involve sums of RVs [1]. Therefore, the evaluation of outage probability $(\mathrm{OP})$ values turns out to be equivalent to computing the cumulative distribution function (CDF) of fading channel envelopes for EGC and of channel gains for MRC [2].

Except for the CDF of the sum of two Rayleigh [3], closedform expressions of the CDF of the sum of fading channel envelopes have not been derived in the literature. To fill this gap, various approximation methods have been instead proposed [4]-[7]. Generally, these approximations are non generic and their accuracy may degrade for a certain choice of the system parameters. Therefore, alternative approaches are of primordial practical interest. One of the alternative is to employ the Monte Carlo (MC) method. However, given the high computational work incurred by this simulation method when small values of the CDF of the sum are considered, this method is unpractical. To avoid such a problem, importance sampling (IS) is known, when appropriately used, to yield very accurate estimates of OP with a few number of runs [8].

The literature is rich of IS approaches dealing with the estimation of tail probabilities of sums of RVs. However, when we consider our framework, i.e. the probability that a sum of RVs is less than a sufficiently small threshold, few works have been developed. For instance, an exponential twisting approach has been proposed in [9] to deal with the CDF of independent and identically distributed (i.i.d) sum of Log-normal variates. The correlated case has been also considered in [10] and [11]. An efficient IS scheme has been also developed to estimate the CDF of the sum of GammaGamma RVs [12]. In [2], two unified IS approaches have been proposed to estimate OP values over generalized fading channels.

Contrary to the evaluation of OP under EGC diversity model, closed-form expressions of OP at the output of MRC diversity receivers are available for many challenging fading environment. This is the case for independent but not necessarily identically distributed (i.n.i.d) Rayleigh fading channels where the expression of OP at the output of MRC receivers is the CDF of the sum of i.n.i.d exponential RVs which is given in [13]. The same observation holds for the correlated Rayleigh case [14]. The i.i.d $\kappa-\mu$ and $\eta-\mu$ fading model are another examples where the values of OP with the MRC scheme are given respectively by the CDF of the squared $\kappa-\mu$ and squared $\eta-\mu$ variates [15]. This observation is behind the main motivation of our work. In fact, we aim to propose an IS estimator of the OP at the output of EGC diversity receivers, i.e. the probability that the sum of fading channel envelopes falls below a given threshold, based on the knowledge of a closed-form expression of the OP with MRC scheme, i.e. the probability that the sum of channel gains is less than a certain threshold. More specifically, the proposed IS scheme is based on sample rejection where the biased probability density function (PDF) is given by the truncation of the underlying PDF over a multidimensional hypersphere with a radius equal to the specified threshold. For sake of illustration, we apply our approach to the i.n.i.d Rayleigh, the correlated Rayleigh with exponential correlation, and the i.i.d Rice fading channels. Note that the correlated Rayleigh case has not been considered by the existing approaches and hence represents one of the relevant contribution of the present work. Moreover, although an estimator of the CDF of the sum of i.i.d Rice variates is developed in [2], it is not clear how sampling according to the 
biased PDF is performed in that work. This constitutes another contribution of the present work where we show that the CDF of the sum of i.i.d Rice variates is easily implemented using our proposed estimator. We provide, for each case, a detailed procedure of how the proposed estimator is implemented and prove that the bounded relative error property, which is one of the desired property in the context of rare event simulation [8], is achieved.

The rest of the paper is organized as follows. In Section II, we present the problem setting and describe the main concept of IS. Section III is devoted to presenting the general idea of the proposed IS estimator. Moreover, we apply, in the same section, our IS estimator to three interesting scenarios. For each scenario, we provide a detailed implementation procedure and prove that the desired property of bounded relative error holds. Finally, a comparison of our estimator with existing estimators as well as naive MC simulations is performed via various numerical results.

\section{Problem Setting}

The instantaneous SNR at the output of $L$-branch EGC diversity receiver is expressed as [2]

$$
\gamma_{e n d}=\frac{E_{s}}{N_{0} L}\left(\sum_{i=1}^{L} R_{i}\right)^{2}
$$

where $\frac{E_{s}}{N_{0}}$ is the SNR per symbol at the transmitter, $L$ is the number of diversity branches, and $R_{i}$ is the channel envelope, i.e. the fading channel amplitude, of the $i^{t h}$ diversity branch. The OP, which is a widely used metric for performance analysis of wireless communication systems operating over fading channels, is defined as the probability that the SNR $\gamma_{e n d}$ is below a given threshold $\gamma_{t h}$

$$
P_{\text {out }}=P\left(\gamma_{\text {end }} \leq \gamma_{t h}\right),
$$

which is equivalent, using the SNR expression in (1), to

$$
P_{\text {out }}=P\left(\sum_{i=1}^{L} R_{i} \leq \gamma_{0}\right)
$$

where $\gamma_{0}=\sqrt{\frac{\gamma_{t h} L N_{0}}{E_{s}}}$. Thus, the problem is reduced to evaluating the CDF of the sum of fading envelopes (modulus of the fading channels) of the $L$ diversity branches. Unfortunately, this quantity is out of reach for many well-practical fading models. A non exhaustive list includes for instance the Rayleigh fading environment where the CDF of the sum of correlated (or even independent) Rayleigh RVs is not known to has a closed-form repression. A similar observation holds also for the independent Rician, the $\kappa-\mu$, and the $\eta-\mu$ fading models.

Naive MC simulations constitute a good alternative to estimate the CDF of the sum of fading envelopes. Let us denote by $f(\cdot)$ the joint PDF of the random vector containing the $L$ fading envelopes $\mathbf{R}=\left(R_{1}, R_{2}, \cdots, R_{L}\right)$. Then, using $M$ independent replicants $\left\{\mathbf{R}^{(k)}\right\}_{k=1}^{M}$ of the random vector $\mathbf{R}$ sampled according to $f(\cdot)$, the naive MC estimator is defined as

$$
\hat{P}_{\text {out }, M C}=\frac{1}{M} \sum_{k=1}^{M} \mathbf{1}_{\left(\sum_{i=1}^{L} R_{i}^{(k)} \leq \gamma_{0}\right)},
$$

where $\mathbf{1}_{(\cdot)}$ denotes the indicator function. However, the high computational complexity, in terms of required number of samples to ensure an accurate estimate, incurred by this method makes it unpractical for sophisticated wireless communication systems wherein $P_{\text {out }}$ is sufficiently small. To illustrate such a point, the naive MC method requires a number of runs approximately equal to $100 / P_{\text {out }}$ to estimate $P_{\text {out }}$ with $20 \%$ relative error.

When appropriately used, IS can save a substantial amount of computational gain compared to naive MC simulations. The idea of IS is to rewrite $P_{\text {out }}=\mathbb{E}_{f}\left[\mathbf{1}_{\left(\sum_{i=1}^{L} R_{i} \leq \gamma_{0}\right)}\right]$, where $\mathbb{E}_{f}[\cdot]$ is the expectation with respect to the PDF $f(\cdot)$, as follows

$$
P_{\text {out }}=\mathbb{E}_{g}\left[\mathbf{1}_{\left(\sum_{i=1}^{L} R_{i} \leq \gamma_{0}\right)} L\left(R_{1}, \cdots, R_{L}\right)\right],
$$

where $g(\cdot)$ is a new PDF named as IS PDF or biased PDF and $\mathbb{E}_{g}[\cdot]$ denotes the expectation operator with respect to the PDF $g(\cdot) . L$ is the likelihood ratio defined as the ratio between the original and the new introduced PDF

$$
L\left(R_{1}, \cdots, R_{L}\right)=\frac{f\left(R_{1}, \cdots, R_{L}\right)}{g\left(R_{1}, \cdots, R_{L}\right)} .
$$

Then, using $M$ samples $\left\{\mathbf{R}^{(k)}\right\}_{k=1}^{M}$ of the random vector $\mathbf{R}$ sampled according to $g(\cdot)$, we construct the IS estimator as follows

$$
\hat{P}_{\text {out }, I S}=\frac{1}{M} \sum_{k=1}^{M} \mathbf{1}_{\left(\sum_{i=1}^{L} R_{i}^{(k)} \leq \gamma_{0}\right)} L\left(R_{1}^{(k)}, \cdots, R_{L}^{(k)}\right) .
$$

The remaining step is in the choice of the biased PDF $g(\cdot)$ that results in a variance reduction and hence in a computational gain with respect to naive $\mathrm{MC}$ simulations. Before that, it is necessary to define some performance metrics to measure the goodness of any IS scheme. Among these criteria, we focus in this work on the bounded relative error property [8]. We say that the estimator $\mathbf{1}_{\left(\sum_{i=1}^{L} R_{i} \leq \gamma_{0}\right)} L\left(R_{1}, \cdots, R_{L}\right)$ has the bounded relative error property when

$$
\limsup _{\gamma_{0} \rightarrow 0} \frac{\operatorname{var}_{g}\left[\mathbf{1}_{\left(\sum_{i=1}^{L} R_{i} \leq \gamma_{0}\right)} L\left(R_{1}, \cdots, R_{L}\right)\right]}{P_{\text {out }}^{2}}<+\infty .
$$

This property has been used for instance in [2] and it means, when it holds, that the number of samples needed to meet a certain accuracy requirement remains bounded regardless of how small $P_{\text {out }}$ is. Hence, it is sufficient to guarantee a substantial amount of computational gain over naive $\mathrm{MC}$ simulations.

\section{SAMPLE REJECTION IS ESTIMATOR}

Before presenting our choice of the biased pdf $g(\cdot)$, it is convenient for the readers understanding to describe the 
optimal IS density which is defined as the truncation of $f(\cdot)$ over the rare set $\left\{\sum_{i=1}^{L} R_{i} \leq \gamma_{0}\right\}$

$$
g^{*}\left(r_{1}, \cdots, r_{L}\right)=\frac{f\left(r_{1}, \cdots, r_{L}\right) \mathbf{1}_{\left(\sum_{i=1}^{L} r_{i} \leq \gamma_{0}\right)} .}{P_{\text {out }}} .
$$

The above optimal IS density, known also as the zero variance measure, is unpractical since the corresponding likelihood ratio is the unknown quantity $P_{\text {out }}$. However, this measure provides some insights on how the IS density may be selected in order to yield a substantial amount of variance reduction. In fact, it encourages samples that belong to the rare set and maintains over it the likelihood ratio constant. That being said, our idea is then to propose a biased PDF that is the truncation of the underlying PDF $f(\cdot)$ over another set $S$

$$
g\left(r_{1}, \cdots, r_{L}\right)=\frac{f\left(r_{1}, \cdots, r_{L}\right) \mathbf{1}_{(\mathbf{R} \in S)}}{\tilde{P}_{\text {out }}},
$$

where $S$ contains the set of interest $\left\{\left(r_{1}, \cdots, r_{L}\right), \sum_{i=1}^{L} r_{i} \leq\right.$ $\left.\gamma_{0}, r_{i} \geq 0\right\}$ and $\tilde{P}_{\text {out }}$ is the probability that the random vector $\mathbf{R}$ is in $S$. Obviously, in order to be able to implement the IS approach with the above biased PDF, the quantity $\tilde{P}_{\text {out }}$ has to be known in a closed-form expression.

It is important to mention that the squared coefficient of variation, defined as the ratio between the variance of an estimator to its squared mean, of the proposed IS estimator is given by

$$
\frac{\operatorname{var}_{g}\left[\mathbf{1}_{\left(\sum_{i=1}^{L} R_{i} \leq \gamma_{0}\right)} L\left(R_{1}, \cdots, R_{L}\right)\right]}{P_{\text {out }}^{2}}=\frac{\tilde{P}_{\text {out }}}{P_{\text {out }}}-1 .
$$

Therefore, in order to ensure a substantial amount of variance reduction, $S$ has to be selected such that $\tilde{P}_{\text {out }}$ is close to $P_{\text {out }}$. More precisely, the closer is $\tilde{P}_{\text {out }}$ to $P_{\text {out }}$ the smaller is the squared coefficient of variation and hence the more efficient is the proposed IS estimator. Particularly, the bounded relative error holds when $\tilde{P}_{\text {out }} / P_{\text {out }}$ is bounded for a sufficiently small threshold. Note that when $S$ is equal to the rare set $\left\{\left(r_{1}, \cdots, r_{L}\right), \sum_{i=1}^{L} r_{i} \leq \gamma_{0}, r_{i} \geq 0\right\}, g(\cdot)$ reduces to the zero variance density $g^{*}(\cdot)$.

Our choice of $S$ follows from the following observation. For many fading models and for MRC receivers, the OP, which is given in this case by the CDF of the sum of squared fading envelopes, is known in closed-form expressions. Therefore, the set $S$ is chosen as follows:

$$
S=\left\{\left(r_{1}, \cdots, r_{L}\right), \sum_{i=1}^{L} r_{i}^{2} \leq \gamma_{0}^{2}, r_{i} \geq 0\right\},
$$

and thus $\tilde{P}_{\text {out }}$ is the OP at the output of MRC receivers which is given by:

$$
\tilde{P}_{\text {out }}=P\left(\sum_{i=1}^{L} R_{i}^{2} \leq \gamma_{0}^{2}\right) .
$$

In other words, based on the knowledge of a closed-form expressions of the OP at the output of MRC receivers, we construct an IS estimator of OP values at the output of EGC diversity receivers. In the next section, we provide more details about the implementation of the above IS scheme for the case of i.n.i.d Rayleigh, correlated Rayleigh, and i.i.d Rice fading channels. Moreover, we perform a theoretical study of the proposed estimator and show that for each cases it achieves the bounded relative error property. We note here that the following case studies are just for illustrations since our approach can be applicable to other scenarios such as i.n.i.d Nakagami-m, i.i.d $\kappa-\mu$, and i.i.d $\eta-\mu$ fading channels.

\section{A. i.n.i.d Rayleigh Fading Channels}

We consider the first case study where $R_{i}, i=1,2, \cdots, L$, follows i.n.i.d Rayleigh distributions. Hence, the PDF $f(\cdot)$ is given by

$$
f\left(r_{1}, \cdots, r_{L}\right)=\prod_{i=1}^{L} f_{R_{i}}\left(r_{i}\right),
$$

where the univariate PDF of $R_{i}$ is given by

$$
f_{R_{i}}(r)=\frac{2 r}{\Omega_{i}} \exp \left(-r^{2} / \Omega_{i}\right), \quad r \geq 0 .
$$

Next, in order to be able to apply our proposed IS approach, it is essential to provide a closed-form expression of the quantity $\tilde{P}_{\text {out }}$. This expression is obtained from [13] as follows:

$$
\tilde{P}_{\text {out }}=1-(1,0, \cdots, 0) \exp \left(\gamma_{0}^{2} \mathbf{A}(\boldsymbol{\Omega})\right)(1,1, \cdots, 1)^{\prime},
$$

with $\boldsymbol{\Omega}=\left(\Omega_{1}, \cdots, \Omega_{L}\right)^{T}$, $\exp \left(\gamma_{0}^{2} \mathbf{A}(\boldsymbol{\Omega})\right)$ denotes the matrix exponential of $\gamma_{0}^{2} \mathbf{A}(\boldsymbol{\Omega})$ and

$\mathbf{A}(\boldsymbol{\Omega})=\left(\begin{array}{ccccc}-1 / \Omega_{1} & 1 / \Omega_{1} & 0 & \ldots & 0 \\ 0 & -1 / \Omega_{2} & 1 / \Omega_{2} & \ldots & 0 \\ \vdots & \vdots & \ddots & \ddots & \vdots \\ 0 & \cdots & 0 & -1 / \Omega_{N-1} & 1 / \Omega_{N-1} \\ 0 & \cdots & 0 & 0 & -1 / \Omega_{N}\end{array}\right)$

In the implementation of the proposed IS estimator, one has to be able to efficiently sample from the biased PDF $g(\cdot)$ given in (10), that is the truncation of the underlying PDF $f(\cdot)$ over the set $S$ given in (12). To do that, we denote by $G_{i}=R_{i}^{2} / \gamma_{0}^{2}, i=1,2, \cdots, L$ and thus our problem reduces to sampling $G_{1}, \cdots, G_{L}$ according to their underlying PDF truncated over the set $\left\{\sum_{i=1}^{L} G_{i} \leq 1\right\}$. To this end, we propose to use the acceptance-rejection technique with a proposal PDF the uniform distribution over the unit simplex $\left\{\sum_{i=1}^{L} G_{i} \leq 1\right\}$. The whole procedure is described in Algorithm 1

\begin{tabular}{l} 
Algorithm 1 Samples Truncated over $S$ independent Rayleigh \\
case \\
1: Inputs: $\left\{\Omega_{i}\right\}_{i=1}^{L}, \gamma_{0}$. \\
2: Outputs: $\left\{R_{i}\right\}_{i=1}^{L}$. \\
3: while $U>\exp \left(-\gamma_{0}^{2} \sum_{i=1}^{N} U_{i} / \Omega_{i}\right)$ do \\
4: Generate $\left\{U_{i}\right\}_{i=1}^{N}$ from the uniform distribution over \\
$\quad$ the set $\left\{u_{i} \geq 0, \sum_{i=1}^{N} u_{i} \leq 1\right\}$, see [8, Algorithm 3.23]. \\
5: Generate $U$ a sample from the uniform distribution over \\
$\quad[0,1]$. \\
6: end while \\
7: $\mathbf{G} \leftarrow \mathbf{U}$ \\
8: Set $R_{i} \leftarrow \gamma_{0} \sqrt{G_{i}}$. \\
\hline
\end{tabular}


Next, we provide a theoretical efficiency result of the proposed IS estimator. In fact, we show in the following proposition that it has a bounded relative error.

Proposition 1. In the case of independent Rayleigh fading channels, the proposed IS estimator of $P_{\text {out }}$ achieves the bounded relative error property, that is

$$
\limsup _{\gamma_{0} \rightarrow 0} \frac{\tilde{P}_{\text {out }}}{P_{\text {out }}}<\infty \text {. }
$$

Proof. We first upper bound the quantity $\tilde{P}_{\text {out }}$ as follows

$$
\begin{aligned}
\tilde{P}_{\text {out }}=P\left(\sum_{i=1}^{L} R_{i}^{2} \leq \gamma_{0}^{2}\right) & \leq P\left(R_{1} \leq \gamma_{0}, \cdots, R_{L} \leq \gamma_{0}\right) \\
& =\prod_{i=1}^{L}\left(1-\exp \left(-\gamma_{0}^{2} / \Omega_{i}\right)\right) .
\end{aligned}
$$

Then, we lower bound $P_{\text {out }}$ to obtain

$$
\begin{aligned}
P_{\text {out }}=P\left(\sum_{i=1}^{L} R_{i} \leq \gamma_{0}\right) & \geq P\left(R_{1} \leq \gamma_{0} / L, \cdots, R_{L} \leq \gamma_{0} / L\right) \\
& =\prod_{i=1}^{L}\left(1-\exp \left(-\gamma_{0}^{2} / L \Omega_{i}\right)\right) .
\end{aligned}
$$

Therefore, we obtain the following result

$$
\frac{\tilde{P}_{\text {out }}}{P_{\text {out }}} \leq \frac{\prod_{i=1}^{L}\left(1-\exp \left(-\gamma_{0}^{2} / \Omega_{i}\right)\right)}{\prod_{i=1}^{L}\left(1-\exp \left(-\gamma_{0}^{2} / L \Omega_{i}\right)\right)}
$$

By applying the limit superior on both side, the proof is concluded.

\section{B. Correlated Rayleigh Fading Channels}

We consider in this part the case where the Rayleigh fading channels are correlated. The correlation model that we adopt is presented in [14]. In fact, we consider two $L$ dimensional Gaussian random vectors $\mathbf{X}$ and $\mathbf{Y}$ with zero means and same covariance matrices $\boldsymbol{\Sigma}$. We assume for simplicity that $\mathbb{E}\left[\mathbf{X} \mathbf{Y}^{T}\right]=0$ (the cross covariance matrix is zero). Let us define the random vector $\mathbf{R}$ as follows

$$
R_{i}=\sqrt{X_{i}^{2}+Y_{i}^{2}}, \quad i=1, \cdots, L .
$$

It is obvious that $\mathbf{R}$ is a multivariate Rayleigh random vector with correlated components. We settle for a particular structure of the covariance matrix $\boldsymbol{\Sigma}$. In fact, we assume that $\boldsymbol{\Sigma}$ is a matrix of exponential correlations, that is

$$
\Sigma_{i j}= \begin{cases}\sigma^{2} & \text { if } i=j \\ \rho^{|i-j|} \sigma^{2} & \text { if } i \neq j\end{cases}
$$

With this structure of the covariance matrix, the multivariate Rayleigh PDF is given by [14]

$$
\begin{aligned}
& f\left(r_{1}, \cdots, r_{L}\right)=\frac{\prod_{i=1}^{L} r_{i}}{\sigma^{2 L}\left(1-\rho^{2}\right)^{L-1}} \\
& \times \exp \left(-\frac{1}{2\left(1-\rho^{2}\right) \sigma^{2}}\left[r_{1}^{2}+r_{L}^{2}+\left(1+\rho^{2}\right) \sum_{i=2}^{L-1} r_{i}^{2}\right]\right) \\
& \times \prod_{i=1}^{L-1} I_{0}\left(\frac{\rho}{\left(1-\rho^{2}\right) \sigma^{2}} r_{i} r_{i+1}\right), \quad r_{1}, r_{2}, \cdots, r_{L} \geq 0,
\end{aligned}
$$

where $I_{0}(\cdot)$ denotes the zero order modified Bessel function of the first kind [16]. Now, we aim to obtain a closed-form expression of $\tilde{P}_{\text {out }}$. In our setting, it was proven in [14, Eq.104] that the moment generating function of $\sum_{i=1}^{L} R_{i}^{2}$ is given by

$$
M_{\sum_{i=1}^{L} R_{i}^{2}}(s)=\frac{1}{\prod_{i=1}^{L}\left(1-2 s \lambda_{i}\right)}, \quad s<\frac{1}{2 \lambda_{i}} \text { for all } i
$$

where $\lambda_{i}, i=1, \cdots, L$ are the eigenvalues of the Gaussian covariance matrix $\Sigma$. Therefore, we deduce that $\sum_{i=1}^{L} R_{i}^{2}$ has the same distribution as the sum of $L$ independent exponential RVs with means $2 \lambda_{i}, i=1,2, \cdots, L$. Hence, the quantity $\tilde{P}_{\text {out }}$ is expressed as

$$
\tilde{P}_{\text {out }}=1-(1,0, \cdots, 0) \exp \left(\gamma_{0}^{2} \mathbf{A}(\mathbf{2} \boldsymbol{\lambda})\right)(1,1, \cdots, 1)^{\prime},
$$

with $\boldsymbol{\lambda}=\left(\lambda_{1}, \cdots, \lambda_{L}\right)^{T}$. The remaining step is then to provide an algorithm in order to sample from the biased PDF $g(\cdot)$. To do that, we proceed as in the previous example by applying the acceptance-rejection technique with a uniform distribution over the unit simplex $\left\{\sum_{i=1}^{L} G_{i} \leq 1\right\}$ as a proposal.

Next, we study the efficiency of the proposed estimator and investigate whether the bounded relative error property holds for this example as well.

Proposition 2. In the case of Rayleigh fading channels with exponential correlations, the proposed IS estimator of $P_{\text {out }}$ achieves the bounded relative error property

$$
\limsup _{\gamma_{0} \rightarrow 0} \frac{\tilde{P}_{\text {out }}}{P_{\text {out }}}<\infty .
$$

Proof. We follow the same steps as in the proof of Proposition 1. In fact, we have

$$
\frac{\tilde{P}_{\text {out }}}{P_{\text {out }}} \leq \frac{P\left(R_{1} \leq \gamma_{0}, \cdots, R_{L} \leq \gamma_{0}\right)}{P\left(R_{1} \leq \gamma_{0} / L, \cdots, R_{L} \leq \gamma_{0} / L\right)} .
$$

Then, we use the following asymptotic result of the multivariate $\mathrm{CDF}$ of the Rayleigh random vector which is given in [17]

$$
P\left(R_{1} \leq \gamma_{0}, \cdots, R_{L} \leq \gamma_{0}\right) \sim C \gamma_{0}^{2 L} \text {, as } \gamma_{0} \rightarrow 0
$$

which concludes the proof. 


\section{C. i.i.d Rician Fading Channels}

The last example that we want to analyze is the case where the $R_{i}, i=1, \cdots, L$ are i.i.d Rician fading channels with common PDF

$$
\begin{aligned}
f_{R_{i}}(r) & =\frac{2 r(K+1)}{\Omega} \exp \left(-K-\frac{K+1}{\Omega} r^{2}\right) \\
& \times I_{0}\left(2 r \sqrt{\frac{K(K+1)}{\Omega}}\right), r \geq 0,
\end{aligned}
$$

where $K$ is the Rice factor and $\Omega=\mathbb{E}\left[R_{i}^{2}\right]$, for all $i \in$ $\{1,2, \cdots, L\}$.

In order to obtain an expression of $\tilde{P}_{\text {out }}$, we use the fact that the sum of i.i.d squared Rician, i.e. equivalently the sum of i.i.d non centered Chi squared RVs, is a squared $\kappa-\mu \mathrm{RV}$ with parameters $\kappa=K$ and $\mu=L$ and average power equal to $\tilde{\Omega}=L \Omega$ [15]. Therefore, the quantity $\tilde{P}_{\text {out }}$ is expressed as

$$
\tilde{P}_{\text {out }}=1-Q_{L}\left(\sqrt{2 K L}, \sqrt{\frac{2(K+1) L}{\tilde{\Omega}}} \gamma_{0}\right),
$$

where $Q_{\mu}(\cdot, \cdot)$ is the generalized Marcum $Q$ function [18].

Note that sampling according to the biased PDF $g(\cdot)$ is performed using exactly the same steps as in Algorithm 1.

We show next that the bounded relative error holds again for the case of i.i.d Rician fading channels.

Proposition 3. In the case of i.i.d Rice fading channels, the proposed IS estimator of $P_{\text {out }}$ achieves the bounded relative error property

$$
\limsup _{\gamma_{0} \rightarrow 0} \frac{\tilde{P}_{\text {out }}}{P_{\text {out }}}<\infty .
$$

Proof. First, the CDF of the Rician fading envelope is given by

$$
P\left(R_{i} \leq \gamma_{0}\right)=1-Q_{1}\left(\sqrt{2 K}, \sqrt{\frac{2(K+1)}{\Omega}} \gamma_{0}\right) .
$$

Then, similarly to the previous proofs, we have

$$
\frac{\tilde{P}_{\text {out }}}{P_{\text {out }}} \leq \frac{\left(P\left(R_{1} \leq \gamma_{0}\right)\right)^{L}}{\left(P\left(R_{1} \leq \gamma_{0} / L\right)\right)^{L}} .
$$

By using the following asymptotic result, see [18],

$$
P\left(R_{i} \leq \gamma_{0}\right) \sim \frac{(K+1) \exp (-K)}{\Omega} \gamma_{0}^{2}, \gamma_{0} \rightarrow 0,
$$

the proof is concluded.

\section{Simulation Results}

In this section, we present some simulations to illustrate the theoretical results. Moreover, we study the efficiency of the proposed estimator with respect to other estimators including the naive $\mathrm{MC}$ one. Before showing the results, it is convenient to define some performance metric that will serve as a measure of efficiency of an estimator. In fact, we define the relative error of the naive MC estimator as the relative half-width of its confidence interval

$$
\epsilon_{M C}=\frac{C \sqrt{P_{\text {out }}\left(1-P_{\text {out }}\right)}}{P_{\text {out }} \sqrt{M}},
$$

where $C$ is the confidence constant chosen to be equal to 1.96 (corresponding to $95 \%$ confidence level). The relative error of the proposed estimator is given using a similar argument by

$$
\begin{aligned}
\epsilon_{I S} & =\frac{C \sqrt{\operatorname{var}_{g}\left[\mathbf{1}_{\left(\sum_{i=1}^{L} R_{i} \leq \gamma_{0}\right)} L\left(R_{1}, \cdots, R_{L}\right)\right]}}{P_{\text {out }} \sqrt{M}} \\
& =\frac{C \sqrt{\frac{\tilde{P}_{\text {out }}}{P_{\text {out }}}-1}}{\sqrt{M}} .
\end{aligned}
$$

The comparison between the different estimators is performed in terms of necessary number of simulation runs in order to meet a fixed accuracy requirement measured by the above quantities. More specifically, we set $\epsilon_{M C}$ and $\epsilon_{I S}$ equal to a fixed value and use (36) and (37) to find the number of simulation runs needed to meet this fixed accuracy requirement.

The comparison is performed for two fading models. In a first experiment, we consider the i.i.d Rayleigh fading channels and we evaluate the OP under EGC using the proposed estimator as well the second estimator of [2] which is based on the use of the hazard rate twisting (HRT) technique. Then, we investigate the efficiency of both estimators using the number of simulation runs required to meet a fixed accuracy level. The same steps are repeated in a second experiment for the correlated Rayleigh fading channels with exponential correlations. For this case, the comparison is made with respect to naive MC estimator since, to the best of the authors' knowledge, the case of correlated Rayleigh has not been investigated by previous estimators.

\section{A. i.i.d Rayleigh Fading Channels}

In Fig. 1, we plot the estimated value of $P_{\text {out }}$ given by naive MC simulations, the HRT method which is the second IS approach in [2], and the proposed estimator in the case of i.i.d Rayleigh fading channels. The plot is a function of the threshold value $\gamma_{t h}$ and for three different values of the number of diversity branches $L$.

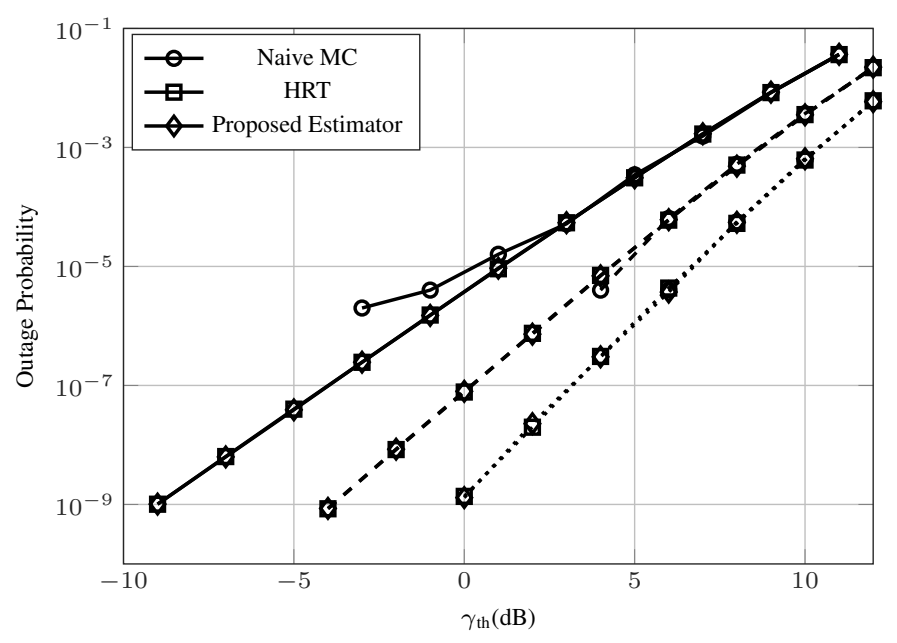

Fig. 1. Outage Probability for $L=4,5,6$ branch EGC receiver with i.i.d Rayleigh fading channels as a function of $\gamma_{t h} . L=4$ (solid line), $L=5$ (dashed line), and $L=6$ (dotted line). The system parameters are $E_{s} / N_{0}=$ $1 \mathrm{~dB}, \Omega=10 \mathrm{~dB}$, and $M=5 \times 10^{5}$. 
This figure reveals the failure of naive MC simulations. In fact, the naive estimator loses its accuracy when the value of $P_{\text {out }}$ decreases, i.e. in the region of rare events. Thus, more than $5 \times 10^{5}$ samples are required in order for the naive sampler to retrieve a good level of accuracy. The inverse observation can be deduced about the proposed estimator and the HRT one. In fact, using the same number of simulation runs, these two estimators coincide perfectly and yield very accurate estimates of $P_{\text {out }}$ in the considered range of OP values.

We investigate now the efficiency of these estimators in terms of number of simulation runs needed to meet a fixed accuracy requirement. More precisely, we compute from (36) and (37) the number of simulation runs needed to ensure that $\epsilon_{M C}=\epsilon_{I S}=\epsilon_{H R T}=5 \%$. Note that $\epsilon_{H R T}$ is given by a similar expression as in (36) and (37). In Fig. 2, we plot the number of samples needed by the naive MC simulation, the proposed method, and the HRT technique as a function of $\gamma_{t h}$ and for the three values of $L$ as in Fig. 1.

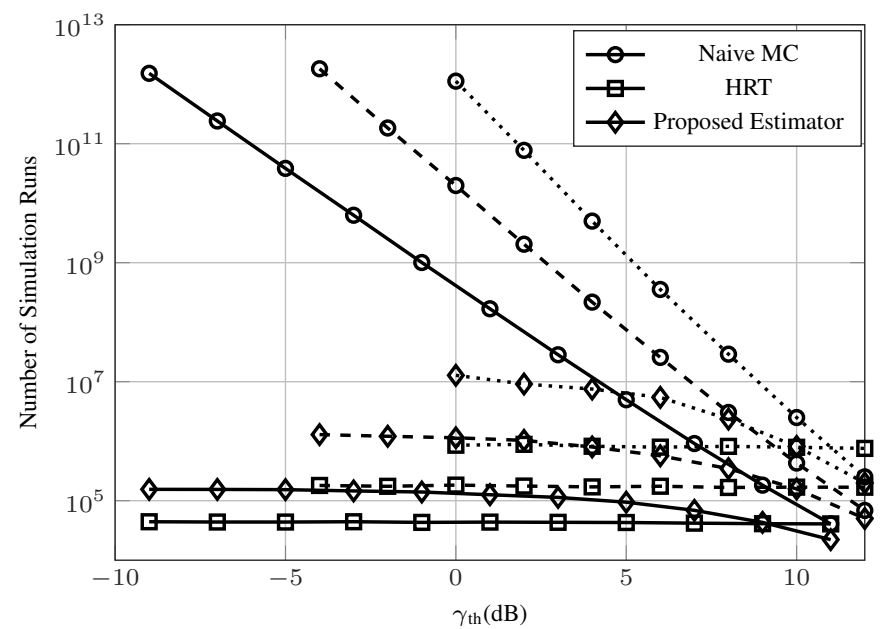

Fig. 2. Number of simulation runs for $L=4,5,6$ branch EGC receiver with i.i.d Rayleigh fading channels as a function of $\gamma_{t h} . L=4$ (solid line), $L=5$ (dashed line), and $L=6$ (dotted line). The system parameters are $E_{s} / N_{0}=1 \mathrm{~dB}$ and $\Omega=10 \mathrm{~dB}$.

We first observe the high computation effort needed by naive MC simulations in order to meet $5 \%$ relative error. In fact, its corresponding number of samples is increasing as we decrease the probability of interest $P_{\text {out }}$. On the other hand, the computational savings of the proposed IS estimator and the HRT one are clearly obvious and are increasing as we decrease $P_{\text {out }}$. More specifically, while the number of samples needed by the naive sampler is increasing as we decrease $\gamma_{t h}$, the proposed IS approach and the HRT method require a number of runs that remains bounded independently of how small $P_{\text {out }}$ is. This observation is in accordance with Proposition 1 and the result proven in [2] which show that both estimators have bounded relative errors. For sake of illustration, for $L=4$ and $\gamma_{t h}=-9 \mathrm{~dB}$, the number of runs needed by naive $\mathrm{MC}$ simulation is approximately $1.5 \times 10^{12}$ whereas $1.5 \times 10^{5}$ and $5 \times 10^{4}$ samples are respectively required by the proposed approach and the HRT estimator to ensure $5 \%$ relative error.

Note also that the HRT approach performs better than our proposed scheme for the considered values of $L$ and $\gamma_{t h}$.
Moreover, Fig. 2 shows that increasing $L$ has negative effects on the performances of the proposed approach as well as the HRT one. However, this negative effect is more important for the former than the latter. For instance, the HRT approach requires 3.5 (respectively 15) times less number of samples than the proposed IS scheme when $P_{\text {out }}$ is of the order of $10^{-9}$ and $L=4$ (respectively $L=6$ ).

Note however that the outperformance of the HRT approach over our proposed one does not tell the whole story and does not exclude the proposed estimator from being a useful technique. In fact, the scope of applicability of the proposed estimator includes the interesting scenario of sums of correlated Rayleigh fading channel with exponential correlations which, to the best of the authors' knowledge, has not been considered by previous estimators. Moreover, while the HRT estimator is proven to have bounded relative error for the sum of i.i.d Rice variate, it is not clear how sampling according to the HRT biased PDF is performed. On the other hand, we showed in Section III-C how our approach can be easily implemented for the i.i.d Rice setting. The same argument holds as well for the sum of i.i.d $\kappa-\mu \mathrm{RVs}$. This again shows the relevance of the proposed estimator. In the following subsection, we apply the proposed estimator to the case of the sum of exponentially correlated Rayleigh RVs and show the high computational saving compared to the naive MC sampler.

\section{B. Correlated Rayleigh Fading Channels}

We consider the case of exponentially correlated Rayleigh fading channels and we aim to perform the same experiment as above. Note that we compare our estimator to the naive $\mathrm{MC}$ one since the authors are not aware of an existing estimator dealing with the CDF of the sum of correlated Rayleigh RVs. In Fig. 3, we plot the estimated value of $P_{\text {out }}$ given by the proposed estimator as well as the naive one as a function of the threshold and for three different values of $L$. The same

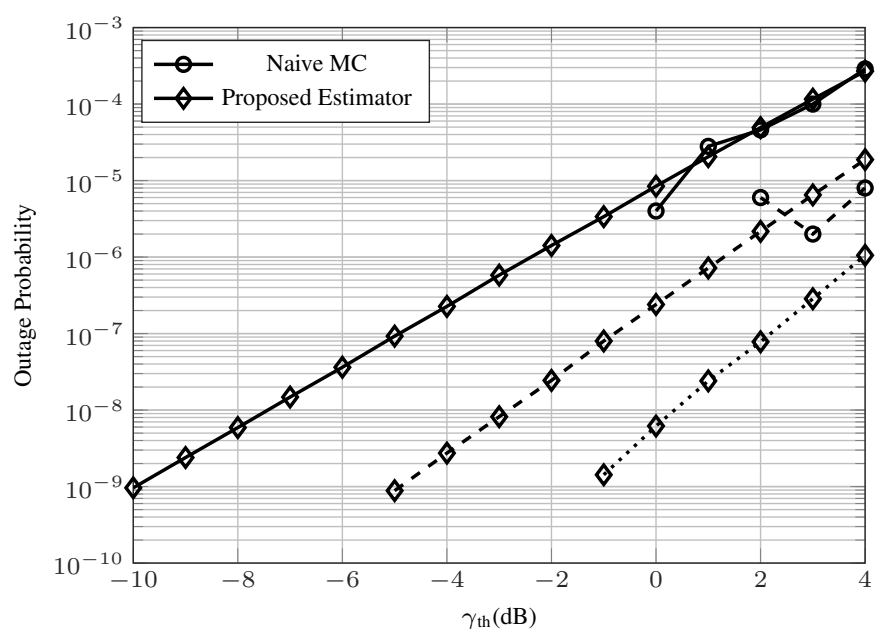

Fig. 3. Outage Probability for $L=4,5,6$ branch EGC receiver with exponentially correlated Rayleigh fading channels as a function of $\gamma_{t h} . L=4$ (solid line), $L=5$ (dashed line), and $L=6$ (dotted line). The system parameters are $E_{s} / N_{0}=1 \mathrm{~dB}, \sigma=\sqrt{5}, \rho=0.5$, and $M=5 \times 10^{5}$.

conclusions can be drawn, as in the previous experiment, about 
the inability of naive MC simulations to yield, using $5 \times 10^{5}$ samples, a precise estimate in the region of small values of $P_{\text {out }}$. On the other side, this number of samples is sufficient for our estimator to provide an estimate of $P_{\text {out }}$ with a good level of accuracy.

Next, we quantify the efficiency of the proposed approach with respect to naive MC simulations in terms of necessary number of simulation runs required to ensure $5 \%$ relative error. This number is plotted in Fig. 4 as a function of $\gamma_{t h}$ using the three values of $L$. We observe the clear outperformance

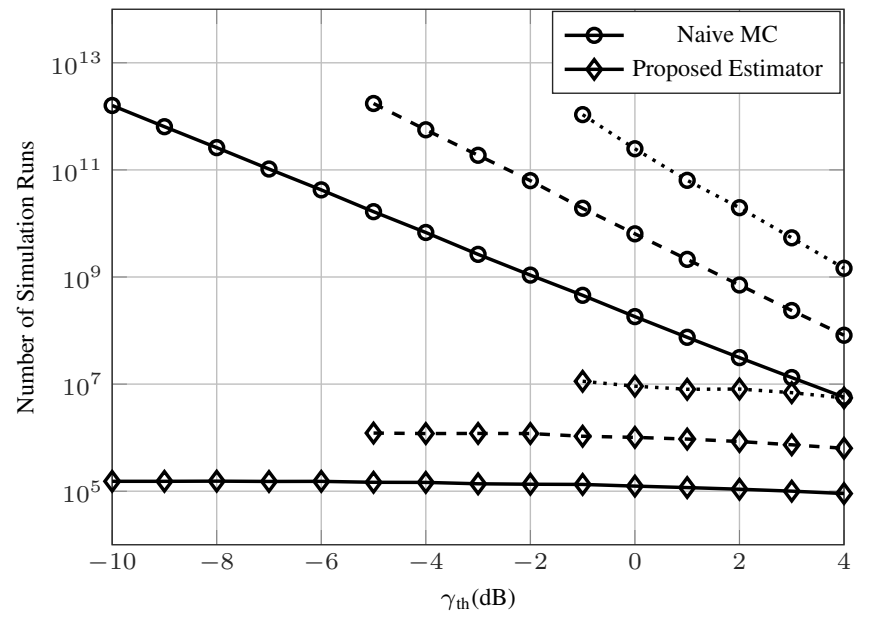

Fig. 4. Number of simulation runs for $L=4,5,6$ branch EGC receiver with exponentially correlated Rayleigh fading channels as a function of $\gamma_{t h}$. $L=4$ (solid line), $L=5$ (dashed line), and $L=6$ (dotted line). The system parameters are $E_{s} / N_{0}=1 \mathrm{~dB}, \sigma=\sqrt{5}$, and $\rho=0.5$.

of the proposed estimator compared to the naive sampler. In fact, contrary to the naive sampler which requires a number of runs that keeps increasing as we decrease the OP values, the number of runs needed by the proposed estimator remains bounded regardless of how much smaller $P_{\text {out }}$ is. This is in accordance with the result proven in Proposition 2. For example, approximately $10^{6}$ simulation runs are needed by the proposed IS estimator when $L=5$ and $\gamma_{t h}$ is less than $-2 \mathrm{~dB}$. On the other hand, the naive sampler requires approximately $10^{11}$ runs (respectively more than $10^{12}$ ) for the same value of $L$ and when $\gamma_{t h}=-2 \mathrm{~dB}$ (respectively when $\gamma_{t h}=-5 \mathrm{~dB}$ ).

\section{CONCLUSiON}

We developed an importance sampling estimator for the estimation of the outage probability at the output of equal gain combining receivers. The proposed biased probability density function is the truncation of the underlying one over the multidimensional sphere. It is based on the perfect knowledge of a closed-form expression of the outage probability with maximum ratio combining receivers. This assumption is not restrictive since it holds for various challenging fading models.
Moreover, we proved for three interesting fading channels that the proposed estimator has bounded relative error. This represents a valuable feature in the field of variance reduction techniques. Finally, we studied through various simulation results the performance of the proposed estimator and compared it with existing ones.

\section{REFERENCES}

[1] M.K. Simon and M.-S. Alouini, Digital Communication over Fading Channels, 2nd Edition. New York: Wiley, 2005.

[2] N. Ben Rached, A. Kammoun, M.-S. Alouini, and R. Tempone, "Unified importance sampling schemes for efficient simulation of outage capacity over generalized fading channels," IEEE Journal of Selected Topics in Signal Processing, vol. 10, no. 2, pp. 376-388, Mar. 2016.

[3] X. Qi, M.-S. Alouini, and Y.-C. Ko, "Closed-form analysis of dualdiversity equal-gain combining over Rayleigh fading channels," IEEE Transactions on Wireless Communications, vol. 2, no. 6, pp. 1120-1125, Nov. 2003.

[4] J. A. Lopez-Salcedo, "Simple closed-form approximation to Ricean sum distributions," IEEE Signal Processing Letters, vol. 16, no. 3, pp. 153 155, Mar. 2009

[5] J. Hu and N. C. Beaulieu, "Accurate simple closed-form approximations to Rayleigh sum distributions and densities," IEEE Communications Letters, vol. 9, no. 2, pp. 109-111, Feb. 2005

[6] M. Di Renzo, F. Graziosi, and F. Santucci, "Further results on the approximation of Log-normal power sum via Pearson type IV distribution: a general formula for log-moments computation," IEEE Transactions on Communications, vol. 57, no. 4, pp. 893-898, Apr. 2009.

[7] C. Tellambura and A. Annamalai, "Further results on the Beaulieu series," IEEE Transactions on Communications, vol. 48, no. 11, pp. 1774-1777, Nov. 2000.

[8] D. P. Kroese, T. Taimre, and Z. I. Botev, Handbook of Monte Carlo methods. N.J: Wiley, 2011.

[9] S. Asmussen, J. L. Jensen, and L. Rojas-Nandayapa, "Exponential family techniques for the Lognormal left tail," Scandinavian Journal of Statistics, vol. 43, no. 3, pp. 774-787.

[10] A. Gulisashvili and P. Tankov, "Tail behavior of sums and differences of Log-normal random variables," Bernoulli, vol. 22, no. 1, pp. 444-493, Feb. 2016.

[11] M.-S. Alouini, N. Ben Rached, A. Kammoun, and R. Tempone, "On the efficient simulation of the left-tail of the sum of correlated Log-normal variates," Monte Carlo Methods and Applications, Mar. 2018.

[12] C. Ben Issaid, N. Ben Rached, A. Kammoun, M. S. Alouini, and R. Tempone, "On the efficient simulation of the distribution of the sum of Gamma-Gamma variates with application to the outage probability evaluation over fading channels," IEEE Transactions on Communications, vol. 65, no. 4, pp. 1839-1848, Apr. 2017.

[13] Z. I. Botev, P. L'Ecuyer, G. Rubino, R. Simard, and B. Tuffin, "Static network reliability estimation via generalized splitting," INFORMS J. on Computing, vol. 25, no. 1, pp. 56-71, Jan. 2013.

[14] R. K. Mallik, "On multivariate Rayleigh and exponential distributions," IEEE Transactions on Information Theory, vol. 49, no. 6, pp. 14991515, Jun. 2003.

[15] M. D. Yacoub, "The $\kappa-\mu$ distribution and the $\eta-\mu$ distribution," IEEE Antennas and Propagation Magazine, vol. 49, no. 1, pp. 68-81, Feb. 2007

[16] I. S. Gradshteyn and I. M. Ryzhik, Table of integrals, series, and products, 7th ed. Elsevier/Academic Press, Amsterdam, 2007.

[17] G. K. Karagiannidis, D. A. Zogas, and S. A. Kotsopoulos, "On the multivariate Nakagami-m distribution with exponential correlation," IEEE Transactions on Communications, vol. 51, no. 8, pp. 1240-1244, Aug. 2003.

[18] S. András, A. Baricz, and Y. Sun, "The generalized Marcum Q- function: an orthogonal polynomial approach," Acta Universitatis Sapientiae

Mathematica, vol. 3, no. 1, pp. 60-76, 2011. 